\title{
The occlusal plane in the facial context: inter-operator repeatability of a new three-dimensional method
}

\author{
Riccardo Rosati, Alberto Rossetti, Marcio De Menezes, Virgilio F Ferrario and Chiarella Sforza
}

The repeatability of a non-invasive digital protocol proposed to evaluate the three-dimensional (3D) position of the occlusal plane in the face is assessed. Dental virtual models and soft tissue facial morphology of 20 adult subjects were digitally integrated using a 3D stereophotogrammetric imaging system. The digital 3D coordinates of facial and dental landmarks were obtained by two different operators. Camper's (facial) and occlusal (dental) planes were individuated, and their 3D relationships were measured. The repeatability of the protocol was investigated and showed no significant differences in repeated digitizations. The angle between occlusal and Camper's planes was smaller than $2^{\circ}$ in the frontal and horizontal projections. In the sagittal projection, the angle was observed to be, on average, $4.9^{\circ}$. The determined occlusal plane pitch, roll and yaw values show good agreement with previously published data obtained by different protocols. The current non-invasive method was repeatable, without inter-operator differences and can facilitate assessment of healthy subjects.

International Journal of Oral Science (2012) 4, 34-37; doi:10.1038/ijos.2012.2; published online 12 January 2012

Keywords: healthy subjects; occlusal plane; stereophotogrammetry; three-dimensional analysis

\section{INTRODUCTION}

The occlusal plane has a key role in several dental and medical disciplines, such as maxillofacial, orthognatic, aesthetic, plastic and reconstructive surgery, general dentistry and prosthodontics. ${ }^{1-3}$

Most dental procedures involve changes in the occlusal plane: orthodontists, maxillofacial surgeons and dental prosthetic professionals could modify its three-dimensional (3D) orientation both in space, and relative to the other craniofacial structures. Several functional aspects are related to the position of the occlusal plane; ${ }^{4-6}$ therefore, the dental professionals should harmonize dental, skeletal and soft tissue structures of the masticatory system.

Several methods have analyzed and measured the craniofacial complex and relative positions of the occlusal and facial planes; most of these investigations used radiographic exams that could detect the relationship between craniofacial structures in a single twodimensional (usually laterolateral) projection. $^{7-9}$ Currently, 3D assessments may be performed overlapping computed tomography reconstruction and digitized dental cast, ${ }^{2,10}$ but the method requires ionizing radiations, and it is not applicable in reference studies performed with healthy subjects. Also, it may be not appropriate to use invasive radiographic protocols in patient requiring nonsurgical-based oral rehabilitations like complete mobile dentures.

Therefore, the physiologic position of the occlusal plane in the coronal and horizontal anatomical planes has not been well defined; a limited number of studies tried to define the 3D orientation of the occlusal plane using non-invasive procedures. ${ }^{11-12}$ These protocols used contact instruments and were based on the recording of the spatial coordinates of a limited set of landmarks of interest. The method did not allow the off-line addition of extra landmarks or the correction of digitization errors. In contrast, optical scanners (laser scanners, stereophotogrammetry units) allow creating data bases of detailed reproductions of facial and dental surfaces permitting a wider choice of future elaborations. Recently, a non-invasive protocol was proposed to reproduce dental and facial surfaces in their correct 3D spatial relationships. ${ }^{13-14}$ In general, the digital 3D protocols of the reproduction of facial and dental surfaces have been found to be reproducible, ${ }^{13-17}$ but no detailed assessments of the $3 \mathrm{D}$ position of the occlusal plane were provided.

The aim of the current study was to assess the inter-operator repeatability of the occlusal plane angular measurements obtained by a new non-invasive protocol. Comparisons with data obtained by different protocols and technology were performed.

\section{MATERIALS AND METHODS}

Experimental design

Healthy subjects were selected to have their maxillary dental casts digitized by laser scanning and their 3D facial soft tissues stereophotogrammetry acquisition merged in a single file. Six landmarks, corresponding to the occlusal and Camper's planes, were selected and exported in a 3D computer-aided design software for a geometrical analysis. The $3 \mathrm{D}$ orientation of the planes, referred to a Cartesian orthogonal reference system, was calculated by two different operators. The reproducibility of the protocol was assessed. The study was in accord with the Declaration of Helsinki, and did not involve invasive or dangerous procedures. 


\section{Subjects}

Twenty subjects ( 11 women and 9 men), aged between 18 and 35 years (average: 25.2 years, s.d.: 4.2) were selected and included in the study. They all were white Caucasians.

Inclusion criteria were: (i) absence of current or recent (in the last 2 years) orthodontic treatment; (ii) no orthognatic surgery history; (iii) no more than three single tooth dental prostheses; (iv) presence of no more than one edentulous socket; and (v) bilateral Angle Class I molar and canine occlusal relationships.

For technical reasons, subjects with beard and moustaches were also excluded. All procedures were not invasive, not dangerous and did not provoke pain or discomfort to the subjects, who were free to stop their examination in any moment. The study protocol was approved by the local ethic committee (12012009-2) and all the analyzed individuals gave their informed consent to the experiment.

\section{Digital dental casts}

For each subject, a maxillary dental reproduction was obtained by an irreversible hydrocolloid (Tropicalgin; Zhermack SpA, Badia Polesine, Italy) and cast with a type 3 model dental stone (Elite model; Zhermack SpA). Using a commercial laser scanner (D100; Imetric $3 \mathrm{D}$, Courgenay, Switzerland), the dental casts were digitized, and the relevant files were imported into the computer-aided design software (see below for details).

\section{Virtual facial reproductions}

Soft tissues facial morphology was acquired by a 3D stereophotogrammetry apparatus (Vectra-3D; Canfield Scientific, Inc., Fairfield, NJ, USA). This imaging system is a modular 3D image capturing system designed to acquire and process stereo images. ${ }^{14,18}$ The system consists of two pods, including three cameras (two black and white, and one color) and a projector in each pod. The system records synchronized pairs of two-dimensional images of the subjects. Using dedicated software, the information was employed to work out the $3 \mathrm{D}$ reconstructions that subsequently can be processed, analyzed, manipulated and measured. The reproducibility of stereophotogrammetric technology was well documented. ${ }^{14,18-20}$

Before each acquisition, three soft-tissue landmarks (Tr, right Tragus; Tl, left Tragus; Sn, Subnasale) were marked on the face of each subject with black liquid eye liner for further analysis. For each subject, two sets of facial 3D images were obtained: one with open lips (with cheek retractors) with visible incisors, canines and premolars, and another one with the teeth in occlusion and closed lips. ${ }^{14}$

\section{Matching}

To obtain virtual dentofacial reproductions, the matching between the 3D facial images and the corresponding maxillary digital dental arch was made using Vectra's software. The digital tools allowed detecting and overlapping common areas in different files. The matching process followed three steps, developed in a previous study and found to be reproducible with limited errors: ${ }^{14}$

- The digital dental cast was merged with the open lips facial acquisition using dental vestibular common surfaces.

- The image with closed lips was introduced into the scene and related to the open lips image overlapping forehead shared areas.

- The open lips acquisition was deleted from the file, obtaining a final digital image with the dental cast and the facial reconstruction with closed lips in the relevant 3D positions.

\section{Evaluated planes}

To investigate the position of the occlusal plane in the facial context, Camper's plane was used as reference. Camper's plane is of easy identification and it is widely used for anthropometric and dental studies involving the position of the dental arch. ${ }^{21-22}$

Camper's plane was defined as passing through right and left Tragus and Subnasale landmarks.

The occlusal plane was identified by three landmarks: interincisal point of the upper central incisors (I) and mesiobuccal cusps of the first upper right and left molars (Mbr and Mbl) (Figure 1a). In both occlusal and Camper's planes, the geometrical sagittal midline was also constructed.

\section{Data measurement}

To obtain an easy data management, the coordinates of the six landmarks of each subject were exported into a 3D computer-aided design software (Rhinoceros 3.0; Robert McNeel \& Associates, Seattle, WA, USA) that allowed to create digital surfaces and to measure angles.

For each subject, the orientation of the occlusal plane was quantified relative to Camper's plane; three geometrical projections were evaluated:

1. Frontal/ coronal projection: angle comprised between the bases of Camper's (Tr-Tl) and occlusal planes (Mbr-Mbl) (Figure 1b).

2. Sagittal projection: angle comprised between the midlines of Camper's and occlusal planes (Figure 1c).

3. Horizontal projection: angle comprised between the midlines of Camper's and occlusal planes (Figure 1d).

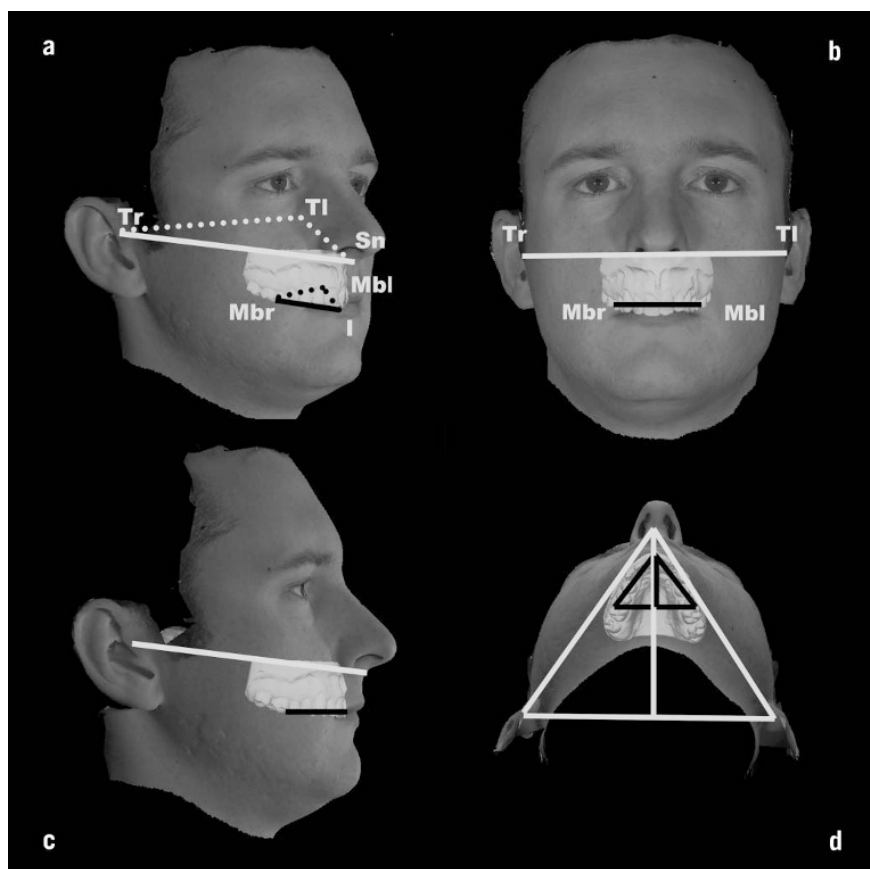

Figure 1 Integration of non-invasive three-dimensional reproductions of hardand soft-tissue facial structures. (a) Camper's plane (white; Tr: right Tragus; TI: left Tragus; Sn: Subnasale); occlusal plane (black; I: inter-incisor; Mbr, Mbl: tip of the mesiovestibular cuspids of right and left first permanent molars) and relevant sagittal midlines. (b) Frontal view. (c) Lateral view with the midlines of Camper's (white) and occlusal (black) planes. (d) Submental view. 


\section{Method error}

The repeatability of the matching procedure between digital facial and dental arch reproductions has previously been reported, with relative errors of measurements up to $1.2 \%$, and without significant differences. ${ }^{14}$ Repeated sets of acquisition of facial landmarks showed interand intra-operator random errors always lower than $1 \mathrm{~mm}$, without systematic biases. ${ }^{18}$

To evaluate the repeatability of the current set of measurements (angles between Camper's and occlusal planes), the protocol was performed independently by two different operators. Altogether 120 measurements were obtained, and imported in a virtual spreadsheet.

Descriptive statistical analysis (mean and standard deviation values) were calculated for each variable. To evaluate the method repeatability, we computed: ${ }^{14,18}$

- mean absolute difference (MAD) between repeated measurements obtained by two different operators, and technical error of measurement (TEM), to assess between operator random errors;

- paired Student's $t$-tests (significance level set at $5 \%, P<0.05$ ), to estimate between-operator systematic differences.

\section{RESULTS}

Overall, the method was reproducible and without systematic biases.

For all three spatial projections, a fair inter-operator repeatability was found, with all MADs and TEMs slightly lower than $2^{\circ}$ (Table 1 ). No systematic errors between repeated measurements were found (Student's $t$-test, $P>0.05$ ).

In the frontal and horizontal projections, the occlusal plane resulted nearly parallel to Camper's plane, with average inclinations of $1.5^{\circ}$ (frontal) and $1.9^{\circ}$ (horizontal). In the sagittal projection, the two planes had an average angle of $4.9^{\circ}$, with the occlusal plane more anteriorly inclined than Camper's plane in 16 subjects of 20 .

In the frontal and horizontal perspectives, respectively, 12 subjects had a right-side oriented occlusal plane, while the others 17 had a leftside oriented occlusal plane.

\section{DISCUSSION}

The base of the restorative, prosthodontic and orthopedic dental treatments is to reestablish a physiological morphology. Quantitative research about the normal anatomical relationships in healthy persons can aid the practitioners during diagnosis and planning of procedures and treatments. Several morphological situations that involve dental-facial relationships can potentially be analyzed by a combination of soft- and hard-tissue assessments.

Currently, X-ray technology allows reproducing the 3D morphology of both hard- and soft-tissue facial structures, ${ }^{2,13,23-24}$ but in a quite invasive way. Indeed, no reference values, or normal morphologic guidelines, could be obtained from non-patient subjects.
The definition of measurement protocols that employ non-invasive procedures may allow the analysis of children, thus permitting to follow up the soft-hard tissue relationships during growth and development. This could aid the practitioner to detect the individual grow spurts, and to perform a more effective treatment. A reliable digital integration of facial soft tissues and dental arches could help clinicians to enforce the aesthetical evaluation during the treatment planning. The use of standardized protocols for a non-invasive facial morphology analysis could replace, at least in part, radiographic assessments. Combined soft-tissue and dental appraisals could be adequate for standard diagnoses and treatment planning in most orthodontic patients and even in the follow-up of surgical patients. ${ }^{25}$

In the current study, a non-invasive protocol for the analysis of dentofacial relationships was defined and evaluated. Overall, the differences between the operators were negligible, with MAD and TEM lower than $2^{\circ}$, and without systematic errors (Student's $t$-tests, $P>0.05$ in all projections).

As tested in a previous investigation, ${ }^{14}$ the precision of the virtual reproductions seems to be satisfactory; the protocol used in the current investigation was appropriate to the initial purpose. Future advances in software implementation should be directed to simplify the protocol, thus allowing its daily use to compare the patient morphology with healthy reference standards. In a recent investigation, Kim et al. ${ }^{2}$ tested the accuracy of matching images of cranial structures obtained by computed tomography and of dental models obtained by laser scanners. The authors employed a procedure similar to the current one, and found negligible errors, but all measurements were made by a single operator. Also Gateno et al. ${ }^{10}$ evaluated the precision of procedures for the integration of dental and skeletal reproductions using extracranial reference points. They found a virtual distortion lower than $1 \mathrm{~mm}$, in good accord with clinical needs. However, they did not test the operator reliability. Therefore, the current investigation widened the previous results showing also a good inter-operator reliability.

To analyze the morphologic results of the $3 \mathrm{D}$ relationships of the occlusal and Camper's planes, the three spatial components should be separately evaluated. In the sagittal projection, no innovative information was obtained; several studies based on lateral radiographic exams, electromagnetic technology or photographic protocols were published. ${ }^{11,25-26}$ In a study of cephalometric radiographs of denture wearers, Nissan et al. ${ }^{27}$ reported that the average angle between the occlusal plane and Camper's line was $7^{\circ}$. In healthy adults of Caucasian origin, Ferrario et al., ${ }^{11}$ using a 3D electromagnetic based technology, photographs ${ }^{26}$ and TV cameras, ${ }^{28}$ reported an average discrepancy between occlusal and Camper's planes in accord with the current results.

In the frontal projection, Camper's and occlusal planes resulted quite parallel, in accord with previous studies. ${ }^{11,26,28}$

Table 1 Differences between angles obtained by two different operators

\begin{tabular}{|c|c|c|c|c|c|c|}
\hline Projection & Operator & Mean degrees & s.d. degrees & MAD degrees & TEM degrees & $P$ value \\
\hline \multirow[t]{2}{*}{ Frontal projection } & 1 & 1.6 & 1.1 & 0.9 & 0.9 & 0.481 \\
\hline & 2 & 1.4 & 0.9 & & & \\
\hline Horizontal projection & 1 & 1.8 & 1.6 & 0.9 & 0.8 & 0.48 \\
\hline \multirow[t]{2}{*}{ Sagittal projection } & 1 & 5.3 & 3.1 & 1.9 & 1.9 & 0.171 \\
\hline & 2 & 4.4 & 2.3 & & & \\
\hline
\end{tabular}

Abbreviations: MAD, mean absolute difference; TEM, technical error of measurement.

$P$ values from paired Student's $t$-tests. 
The use of 3D digital reproductions allowed the assessment of the horizontal plane prospective. In the analyzed subjects, the midlines of Camper's and occlusal planes were almost parallel, with an average difference of $2^{\circ}$. This information may be a key concept in the definition of the dental disposition during a full arch maxillary rehabilitation or orthodontic treatment. In fact, while the classical occlusal evaluation assesses mainly the anteroposterior relationships, a 3D comprehension should be reached. As underlined by Ackerman et $a l .{ }^{29}$ the analysis of the orientations of both the functional line of occlusion and the aesthetic line of the dentition should be best described by a 3D approach, assessing pitch, roll and yaw. Therefore, the definition of the 'yaw' of the dental arches of healthy subjects could be useful to assess biological variability and to define morphological guidelines. Future studies should be encouraged to investigate the horizontal disposition of the occlusal plane in the facial context with a larger sample.

The current study has some technical limitations. From a methodological point of view, the described protocol for the reproduction of the soft-hard tissue relationships requires expert operators. The main technical complication was due to tooth translucence allied with saliva biofilm. In fact, unsatisfactory virtual tooth reconstructions were obtained if a dry tooth surface was not correctly maintained. Another limitation of this protocol might be the cost and availability of the technical instruments for a private dental office, even if the collaboration between research centers and private structures should be encouraged at all levels.

\section{CONCLUSION}

Within the limitation of the current study, the present non-invasive method was appropriate to the aims; no operator's biases were found. In the analyzed subjects, on the sagittal projection, a mean discrepancies of $5^{\circ}$ between occlusal and Camper's planes was measured. The occlusal plane was parallel to Camper's plane in the frontal and horizontal projections. No 'roll or yaw' of maxillary dental arch in a facial context was demonstrated.

1 Jayachandran S, Ramachandran CR, Varghese R. Occlusal plane orientation: a statistical and clinical analysis in different clinical situations. Int J Prosthodont 2008; 17(7): 572-575.

2 Kim BC, Lee CE, Park W et al. Integration accuracy of digital dental models and 3dimensional computerized tomography images by sequential point- and surfacebased markerless registration. Oral Surg Oral Med Oral Pathol Oral Radiol Endod 2010; 110(3): 370-378.

3 Santana-Penin UA, Mora MJ. The occlusal plane indicator: a new device for determining the inclination of the occlusal plane. J Prosthet Dent 1998; 80(3): 374-375.

4 Ogawa T, Koyano K, Suetsugu T. The relationship between inclination of the occlusal plane and jaw closing path. J Prosthet Dent 1996; 76(6): 576-580.

5 Ogawa T, Koyano K, Suetsugu T. Correlation between inclination of occlusal plane and masticatory movement. J Dent 1998; 26(2): 105-112.

6 Sato M, Motoyoshi M, Hirabayashi M et al. Inclination of the occlusal plane is associated with the direction of the masticatory movement path. Eur J Orthodont 2007; 29(1): 21-25.
7 Bassi F, Deregibus A, Previgliano V et al. Evaluation of the utility of cephalometric parameters in constructing complete denture. Part I: Placement of posterior teeth. $J$ Oral Rehabil 2001; 28(3): 234-238.

8 D'Souza NL, Bhargava K. A cephalometric study comparing the occlusal plane in dentulous and edentulous subjects in relation to the maxillomandibular space. J. Prosthet Dent 1996; 75(2): 177-182.

9 Wei R, Claes $\mathrm{P}$, Walters $\mathrm{M}$ et al. Augmentation of linear facial anthropometrics through modern morphometrics: a facial convexity example. Aust Dent J 2011; 56(2): 141-147

10 Gateno J, Xia J, Teichgraeber JF et al. A new technique for the creation of a computerized composite skull model. J Oral Maxillofac Surg 2003; 61(2): 222-227.

11 Ferrario VF, Sforza C, Serrao G et al. A direct in vivo measurement of the threedimensional orientation of the occlusal plane and of the sagittal discrepancy of the jaws. Clin Orthodont Res 2000; 3(1): 15-22.

12 Sforza C, Peretta R, Grandi G et al. Soft tissue facial planes and masticatory muscle function in skeletal Class III patients before and after orthognathic surgery treatment. J Oral Maxillofac Surg 2008; 66(4): 691-698.

13 Rangel FA, Maal TJ, Berge SJ et al. Integration of digital dental casts in 3-dimensiona facial photographs. Am J Orthodont Dentofac Orthop 2008; 134(6): 820-826.

14 Rosati R, de Menezes M, Rossetti A et al. Digital dental cast placement in 3dimensional, full-face reconstruction: a technical evaluation. Am J Orthodont Dentofac Orthop 2010; 138(1): 84-88.

15 Santoro M, Galkin S, Teredesai M et al. Comparison of measurements made on digital and plaster models. Am J Orthodont Dentofac Orthop 2003; 124(1): 101-105.

16 Quimby ML, Vig KW, Rashid RG et al. The accuracy and reliability of measurements made on computer-based digital models. Angle Orthod 2004; 74(3): 298-303.

17 Cha BK, Lee JY, Jost-Brinkmann PG et al. Analysis of tooth movement in extraction cases using three-dimensional reverse engineering technology. Eur J Orthodont 2007; 29(4): 325-331.

18 de Menezes M, Rosati R, Ferrario VF et al. Accuracy and reproducibility of a 3 dimensional stereophotogrammetric imaging system. J Oral Maxillofac Surg 2010; 68(9): 2129-2135.

19 Ayoub A, Garrahy A, Hood C et al. Validation of a vision-based, three-dimensional facial imaging system. Cleft Palate Craniofac J 2003; 40(5): 523-529.

20 Aldridge K, Boyadjiev SA, Capone GT et al. Precision and error of three-dimensional phenotypic measures acquired from 3dMD photogrammetric images. Am J Med Genet Part A 2005; 138(3): 247-253.

21 Fu PS, Hung CC, Hong JM et al. Three-dimensional analysis of the occlusal plane related to the hamular-incisive-papilla occlusal plane in young adults. J Oral Rehabil 2007; 34(2): 136-140.

22 Quran FA, Hazza'a A, Nahass NA. The position of the occlusal plane in natural and artificial dentitions as related to other craniofacial planes. J Prosthodont 2010; 19(6): 601-605.

23 Swennen GR, Mollemans W, Schutyser F. Three-dimensional treatment planning of orthognathic surgery in the era of virtual imaging. J Oral Maxillofac Surg 2009; 67(10): 2080-2092.

24 Macdonald R. Digital imaging for dentists. Aust Dent J 2001; 46(4): 301-305.

25 Ferrario VF, Serrao G, Ciusa V et al. Cephalometric and in vivo measurements of maxillomandibular anteroposterior discrepancies: a preliminary regression study. Angle Orthod 2002; 72(6): 579-584.

26 Ferrario VF, Sforza C, Miani A Jr et al. Craniofacial morphometry by photographic evaluations. Am J Orthodont Dentofac Orthop 1993; 103(4): 327-337.

27 Nissan J, Barnea E, Zeltzer C et al. Relationship between occlusal plane determinants and craniofacial structures. J Oral Rehabil 2003; 30(6): 587-591.

28 Ferrario VF, Sforza C, Tartaglia G et al. New television technique for natural head and body posture analysis. Cranio 1995; 13(4): 247-255.

29 Ackerman JL, Proffit WR, Sarver DM et al. Pitch, roll, and yaw: describing the spatial orientation of dentofacial traits. Am J Orthodont Dentofac Orthop 2007; 131(3): 305 310.

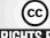

This work is licensed under a Creative Commons Attribution-NonCommercial-NoDerivative Works 3.0 Unported License. To view a copy of this license, visit http:// creativecommons.org/licenses/by-nc-nd/3.0 